MATEC Web of Conferences 41, 03003 (2016)

DOI: $10.1051 /$ matecconf/20164103003

(C) Owned by the authors, published by EDP Sciences, 2016

\title{
Design and development of a hybrid preclinical PET/SPECT/X-ray system
}

\author{
Maria Georgiou, ${ }^{1, a}$, Eleftherios Fysikopoulos ${ }^{1}$, Kostas Mikropoulos ${ }^{1}$, Lazaros Palamaris ${ }^{1}$, Eirini Fragogeorgi ${ }^{1}$ and George \\ Loudos $^{1}$
}

${ }^{1}$ Department of Biomedical Engineering, Technological Educational Institute of Athens, Athens, Greece

\begin{abstract}
In this work we present the design and development of a prototype SPECT/PET/CT system, suitable for whole body small animal imaging, which is unique not only in national but also in regional level. The integrated SPECT/PET/CT system has three components and is mounted on a rotating gantry, which is designed specifically for such applications. The system is unique on national and regional level. The SPECT component has a $2 \mathrm{~mm}$ spatial resolution, the PET components a $2.5 \mathrm{~mm}$ spatial resolution and the X-ray component was recently purchased and is now mounted on the existing gantry and will be evaluated. The complete design of the system and evaluation results are presented.
\end{abstract}

\section{Introduction}

Multimodal molecular imaging is considered as the optimal technology to study non-invasively the biodistribution of new biomolecules, as well as understand molecular mechanisms [1]. While many imaging systems are commercially available, their cost does not allow their purchase by many small and medium research groups. For this reason, several teams build their own prototypes, which are not only used in preclinical studies, but are also exploited as test platforms to assess new detector components and acquisition strategies.

Over the past decade our group has been focused on scintigraphic small animal imaging services and on the development of dedicated imaging systems for such applications [2], [3]. Our research included the evaluation of new radiopharmaceuticals and nanoparticles for controlled drug delivery [4]. However, the continuous interest in PET imaging motivated us to move towards PET imaging. Moreover, the recent trend in molecular imaging is the combination of functional images (SPECT/PET) with anatomical images, in order to localize the hot spots that nuclear images provide. For this reason the addition of an X-ray component on the system could substantially improve its performance and extend its preclinical applications. In this work we present the design and development of a prototype SPECT/PET/CT system, suitable for whole body small animal imaging.

\section{Methods and materials}

The integrated SPECT/PET/CT system will be mounted on an existing rotation station, which is designed specifically for such applications. Figure 1 illustrates the

\footnotetext{
a Corresponding author: mgeorgiou@betsolutions.gr
}

gantry in its current form. The gantry is controlled by a computer, allows bed movement and rotation of the detectors. Its length allows the placement of the SPECT head, the 2 PET heads and the X-ray tube and detector. The animal can move along this axis and perform different types of imaging.

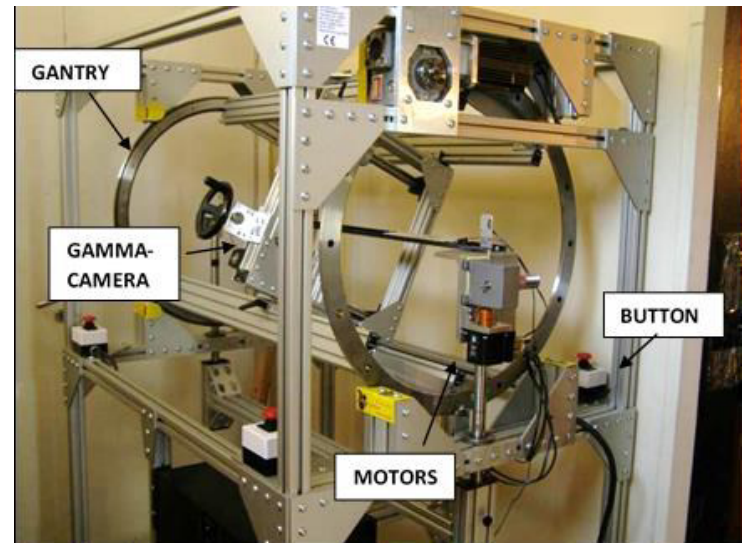

Figure 1. Gantry that supports the SPECT/PET/CT components.

\subsection{SPECT camera}

The existing gamma camera has been replaced by a custom gamma camera, with higher sensitivity, allowing faster dynamic images. It is based on 2x H8500 PSPMTs (Hamamatsu), a $\operatorname{CsI}(\mathrm{Na})$ pixelated scintillator with $1.5 \mathrm{~mm} \times 1.5 \mathrm{~mm} \times 5 \mathrm{~mm}$ pixels and a general purpose hexagonal collimator. The data acquisition system (DAQ) consists of a FPGA (Xilinx SP605) for triggering and digital processing of the pulses acquired using free running ADCs $(40 \mathrm{MHz})$. The evaluation of the system was carried out using phantoms for measuring spatial 
resolution, sensitivity to concentration variations as well as image uniformity. Finally, small animal images were obtained to verify it under real experimental conditions.

In Figure 2, from up left to bottom right the major components of the system are shown including the camera head and power supply, the FPGA, ADC and clock, the two PMTs with analog electronics the collimator and the scintillator.

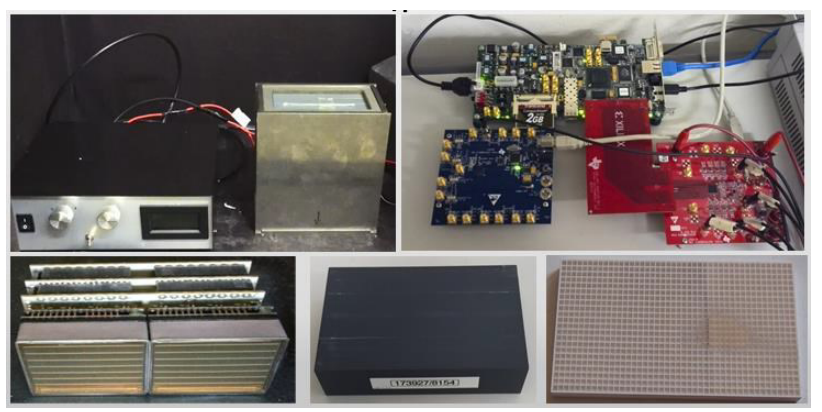

Figure 2. SPECT head and its major components.

\subsection{PET camera}

The PET camera is based on the same PSPMTs coupled to BGO pixelated scintillators with $2 \mathrm{~mm} \times 2 \mathrm{~mm} \times 5 \mathrm{~mm}$ pixel size. The DAQ consists of a bigger FPGA (Spartan $6 \mathrm{LX} 150 \mathrm{~T}$ ) and the sampling rate of the ADCs is $65 \mathrm{MHz}$. The position, the energy and the timestamp of each event are sent to a standard pc via Ethernet link. Reconstruction software has been developed. The PET system has been evaluated in terms of spatial resolution, energy resolution and animal studies have been planned.

\subsection{X-ray detector}

The X-Ray is a high speed, low noise and high sensitivity flat panel detector, model C10900-40 from Hamamatsu. It is based on the CMOS technology and it is suitable for real-time static and CT imaging with high dynamic range due to its switchable pixel gain. The X-ray tube, SourceBlock ${ }^{\mathrm{TM}}$ by Source-Ray, Inc. is a modern technology that integrates an Xray tube and a high voltage generator into one physical package. The SB-80500 model has a 33 micron minimal focal spot, a variable voltage $35-80 \mathrm{kVp}$ and 20 to $500 \mathrm{uA}$ current. The system is currently been mounted on the gantry and then will be evaluated using phantoms and finally small mice.

\section{Results}

\subsection{SPECT camera}

The experimental results showed that the system sensitivity is $130 \mathrm{cps} / \mathrm{MBq}$, which is sufficient for small animal imaging applications. The energy resolution is $26 \%$, although this value can be improved depending on the crystal provider and treatment. The spatial resolution (FWHM) was measured $2.4 \mathrm{~mm}$ on the collimator surfaced and $3.8 \mathrm{~mm}$ at $3 \mathrm{~cm}$ from the collimator.
In Figure 3, 2 capillaries filled in with $\mathrm{Tc}^{99 \mathrm{~m}}$ at $2.8 \mathrm{~mm}$ distance, placed at $7 \mathrm{~mm}$ from the collimator surface and a typical profile, are shown, demonstrating the ability of the system to provide resolution close to $2 \mathrm{~mm}$. On the right part of the image 4 different $\mathrm{Tc}^{99 \mathrm{~m}}$ sources with activity ratios of $1,0.52,0.34$ and 0.13 are shown. The relative intensities are represented on the final image. Quantitative analysis has shown that this information can be obtained even if the acquisition time drops to 10 secs. This very useful information proves that the system is suitable for fast dynamic studies in mice, were the statistics are rather limited.

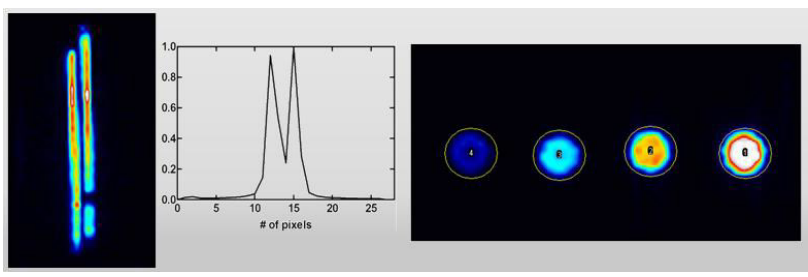

Figure 3. Typical images and line profile of two capillaries filled in with $\mathrm{Tc}^{99 \mathrm{~m}}$ at $2.8 \mathrm{~mm}$ distance placed at $7 \mathrm{~mm}$ from the collimator and four Tc99m sources with activity ratios of 1 , $0.52,0.34$ and 0.13 .

Finally the ability of the system to image small mice with high resolution is shown in Figure 4, where a 30min bone study in a small mouse injected with $\mathrm{Tc}^{99 \mathrm{~m}}-\mathrm{MDP}$ is shown. Clear visualization of mouse bones is shown.

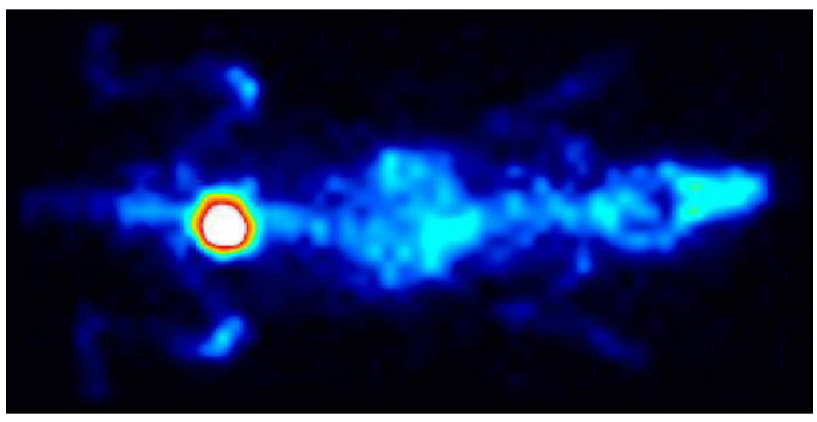

Figure 4. A 30min bone study in a small mouse injected with $\mathrm{Tc}^{99 \mathrm{~m}}$-MDP.

\subsection{PET camera}

Initially the saturation of the system was compared against the coincidence and energy window selection. In all cases the saturation of the system is achieved at $\sim 80 \mathrm{uCi}$, which is an acceptable injected activity for mice. The $20 \mathrm{nsec}$ and $350-700 \mathrm{keV}$ windows were selected as a good compromise between sensitivity and noise.

The imaging ability was measured using a capillary phantom, as it is shown in Figure 6. The results show that energy resolution was $29 \%$, average spatial resolution at planar mode $4.2 \mathrm{~mm}$ (which is improved at 2.5 if tomographic reconstruction is performed), timing resolution of $8.2 \mathrm{nsec}$ and absolute sensitivity at the center of the field of view $0.6 \%$. In all measurements the distance between the two heads was fixed at $5 \mathrm{~cm}$. 


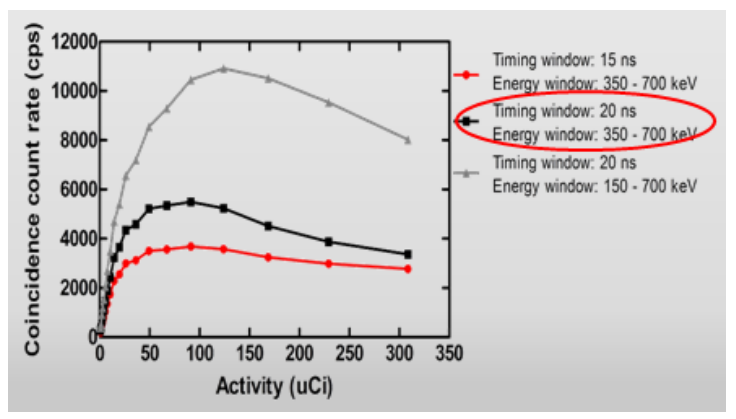

Figure 5. Study of PET system saturation at different windows.

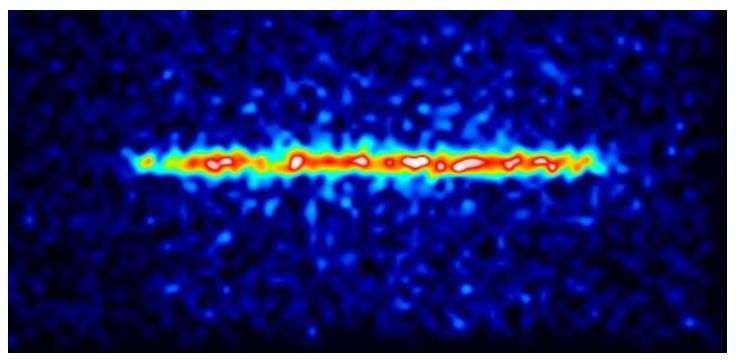

Figure 6. Typical coincidence image of a thin capillary filled with FDG.

To optimize the parameters of tomographic reconstruction, the system was simulated using GATE toolkit [5]. The simulation is shown in Figure 7.

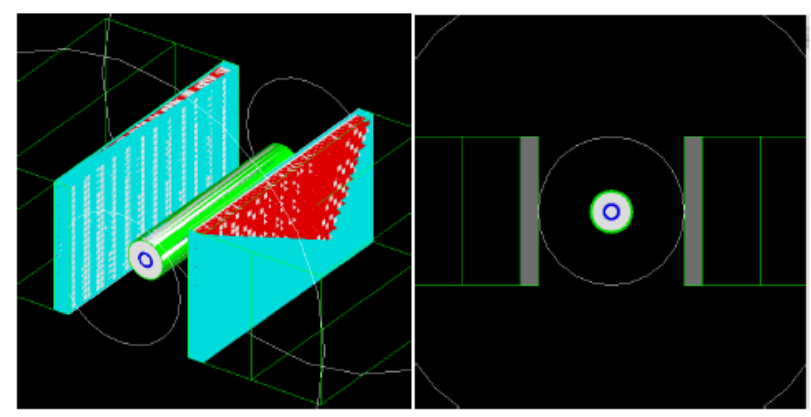

Figure 7. GATE simulation of the PET heads.

Following simple validation tests, more complicated sources were simulated and tomographic reconstruction was carried out by rotating the detectors and acquiring coincidence images from different angles. The results from simulating to custom phantoms of cold and hot cylinders are shown in Figure 8.
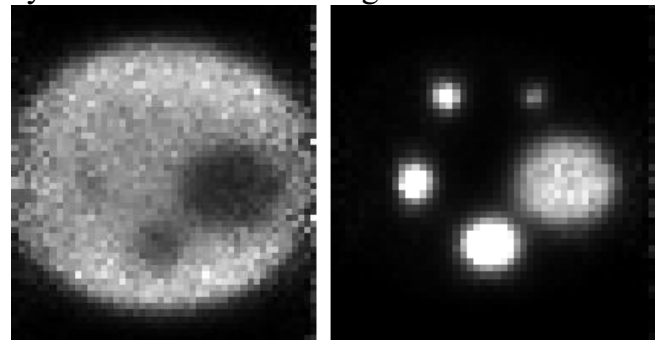

Figure 8. Simulated reconstructed images of cold and hot sources using FDG and imaged with the PET prototype.
The presented results demonstrate that the presented multimodal system can cover the two major imaging modalities of nuclear medicine, namely SPECT and PET. Taking into account that the system is completely open, all its components can be further optimized, in order to improve its performance.

Currently the main focus of the authors is on the evaluation of the X-ray components. In addition the optimization of PET protocols, in order to identify the best parameters for tomographic imaging are studied. The goal of the authors is to implement tomographic algorithms where limited or even no rotation of the PET heads will provide tomographic data. A set of collimators has been designed and ordered, in order to allow imaging of higher energy SPECT isotopes including In-111, Lu177, I-131 and Ga-67. These collimators will be experimentally validated, since the use of higher energy isotopes may introduce changes to the data acquisition hardware.

Further developments and optimization in reconstruction software are expected to improve systems resolution and sensitivity. The addition of a second SPECT head will improve its sensitivity, while the addition of two extra PET heads will have the same effect for the PET component. Finally the rotation of the X-ray component will allow the acquisition of CT data and will allow the exploitation of the system in a new field of applications.

\section{Acknowledgment}

This research has been co-funded by the European Union (European Social Fund) and Greek national resources under the framework of the "ARISTEIA II" project HYPERGNOSTIC code 4309 of the "Education \& Lifelong Learning" Operational Programme.

\section{References}

1. G. Kagadis et al., Med Phys. 37(12), 6421-42 (2010).

2. G. Loudos et al., NIMA 571,48-51 (2007).

3. M. Georgiou et al., 5th Panhellenic Conf. Biomed. Engineering, 4-6 April, (2013)

4. George Loudos et al, Eur J Radiol. 78(2), 287-295 (2011).

5. Sarut et al, Med. Phys. 41, (2014).

\section{Discussion}

